\title{
Suppression of sirtuin 1 alleviates airway inflammation through mTOR-mediated autophagy
}

\author{
YUANYUAN WU, WEI LI, YIFAN HU, YUN LIU and XIUZHEN SUN \\ Department of Respiratory and Critical Care Medicine, The Second Affiliated Hospital of Medical College, \\ Xi'an Jiaotong University, Xi'an, Shaanxi 710004, P.R. China
}

Received August 1, 2019; Accepted April 15, 2020

DOI: $10.3892 / \mathrm{mmr} .2020 .11338$

\begin{abstract}
Sirtuin 1 (SIRT1) is involved in the pathogenesis of allergic asthma. This study aimed to investigate whether EX-527, a specific SIRT1 inhibitor, exerted suppressive effects on allergic airway inflammation in mice submitted to ovalbumin (OVA) inhalation. In addition, this study assessed whether such a protective role was mediated by autophagy suppression though mammalian target of rapamycin (mTOR) activation. Female C57BL/6 mice were sensitized to OVA and EX-527 $(10 \mathrm{mg} / \mathrm{kg})$ was administered prior to OVA challenge. The study found that EX-527 reversed OVA-induced airway inflammation, and reduced OVA-induced increases in inflammatory cytokine expression, and total cell and eosinophil counts in bronchoalveolar lavage fluid. In addition, EX-527 enhanced mTOR activation, thereby suppressing autophagy in allergic mice. To assess whether EX-527 inhibited airway inflammation in asthma through the mTOR-mediated autophagy pathway, rapamycin was administered to mice treated with EX-527 after OVA sensitization. All effects induced by EX-527, including increased phosphorylated-mTOR and decreased autophagy, were abrogated by rapamycin treatment. Taken together, the present findings indicated that EX-527 may inhibit allergic airway inflammation by suppressing autophagy, an effect mediated by mTOR activation in allergic mice.
\end{abstract}

\section{Introduction}

Allergic asthma is one of the most common chronic inflammatory disorders characterized by airway hyperresponsiveness and increased infiltration of inflammatory cells, especially eosinophils, into the airways and lungs (1). The T helper

Correspondence to: Dr Xiuzhen Sun or Dr Yun Liu, Department of Respiratory and Critical Care Medicine, The Second Affiliated Hospital of Medical College, Xi'an Jiaotong University, 157 West 5th Road, Xi'an, Shaanxi 710004, P.R. China

E-mail: doc-ly@sohu.com

E-mail: yangkun113@sohu.com

Key words: asthma, sirtuin 1, airway inflammation, autophagy, mammalian target of rapamycin cell (Th)2 immune response and associated cytokines, such as IL-4, IL-5 and IL-13, are known to play important roles in the pathogenesis of asthma (2).

A total of seven sirtuin (SIRT) family members have been identified in mammals, termed SIRT1 to SIRT7 (3). SIRT1 and SIRT2 are found in the nucleus and cytoplasm, whereas SIRT3, SIRT4 and SIRT5 are mitochondrial, and SIRT6 and SIRT7 are exclusively nuclear (4). SIRT1 is a NAD ${ }^{+}$-dependent class III histone deacetylase protein, which performs a wide variety of functions in biological systems, with implications in metabolic diseases, cancer and inflammation $(5,6)$. SIRT1 regulates various cellular processes, including inflammation, chromatin stability and oxidative stress, by deacetylating nuclear factor- $\kappa \mathrm{B}$, forkhead box protein $\mathrm{O} 3$ and hypoxia-inducible factor $1 \alpha$ (7). Multiple studies have previously suggested that SIRT1 may be involved in the pathogenesis of allergic airway inflammatory diseases, such as asthma $(8,9)$. It was previously reported that SIRT1 inhibition significantly reduced airway hyperresponsiveness, inflammatory cell infiltration into the airways, and increased levels of IL-4, IL-5 and IL-13 after OVA inhalation (8). Furthermore, serum SIRT1 levels were revealed to be significantly elevated in patients with asthma and OVA-induced allergic mice, and were positively correlated with total serum IgE levels and were negatively correlated with pulmonary function (9). However, the roles of SIRT1 in asthma remain largely unclear.

Rapamycin represents a macrolide with known immunosuppressive functions (10). While rapamycin affects multiple nutrient signaling-associated pathways, it acts mainly as a mammalian target of rapamycin (mTOR) inhibitor, by directly suppressing mTOR complex 1 (11). mTOR, an evolutionarily conserved serine/threonine kinase, is a central regulator of cell transcription, growth, proliferation and survival (12). Previous studies have reported that mTOR signaling is involved in inflammatory diseases, including dermatological ailments (13), chronic obstructive pulmonary disease $(14,15)$ and asthma (16). Furthermore, it was revealed that autophagy may be involved in the regulation of asthma $(17,18)$, and enhanced autophagy was reported to be associated with increased asthma severity (18). Autophagy is controlled by the mTOR signaling pathway (19). In allergic mice, the lung levels of phosphorylated (p)-mTOR were decreased, whereas mTOR signaling activation inhibited allergic airway inflammation by suppressing autophagy (16). Additionally, the mTOR and 
SIRT1 signaling pathways are closely integrated and play an important role in the neuroprotective effect of caloric restriction (20). A previous study indicated that SIRT1 induced the growth and survival of neurons in the central nervous system though negative modulation of mTOR signaling (21). Therefore, it is possible that the mTOR and SIRT1 signaling pathways interact to modulate autophagy in asthmatic mice.

It has been reported that serum SIRT1 levels are increased in patients with asthma and OVA-induced allergic mice, whereas SIRT1 inhibition ameliorates airway inflammation in allergic mice. However, the mechanism underlying allergic airway inflammation suppression is unclear. EX-527 is a well-known specific inhibitor of SIRT1 $(22,23)$, selective inhibition of SIRT1 by EX-527 could alleviate endotoxemia-associated acute lung injury (22). Therefore, the aim of this study was to investigate the effect and mechanism of EX-527 on allergic airway inflammation in a murine model of asthma.

\section{Materials and methods}

Animals and experimental design. A total of 32 female C57BL/6 mice (age, 6-8 weeks; weight, 20-22 g) were purchased from the Experimental Animal Center Medical College of Xi'an Jiaotong University (Xi'an, China). All animals were maintained in steel cages in a room with controlled temperature at $23-25^{\circ} \mathrm{C}$, under a 12 -h light/dark cycle in a specific pathogen-free facility, with access to food and water ad libitum. All experimental protocols were approved by the medical ethics committee of The Second Affiliated Hospital of Xi'an Jiaotong University. Mice were maintained in an animal facility under standard laboratory conditions for 1 week prior to experiments, and were randomly assigned into four groups ( $\mathrm{n}=8 / \mathrm{group}$ ): i) Control, ii) OVA, iii) EX-527 and iv) rapamycin groups. The OVA group was sensitized by subcutaneous injection of OVA $(50 \mu \mathrm{g} / \mathrm{kg})$ adsorbed to $2 \mathrm{mg}$ aluminum hydroxide in $200 \mu \mathrm{l}$ normal saline at 0,7 and 14 days, then challenged by exposure to an aerosol containing 1\% OVA (w/v) in PBS for 30 min using an ultrasonic nebulizer (Soniclizer305, ATOM) on days 21, 23, 25 and 27 after the initial sensitization. In the EX-527 group, mice received EX-527 administration $(10 \mathrm{mg} / \mathrm{kg})$ by intraperitoneal injection $1 \mathrm{~h}$ prior to OVA inhalation. The control group mice were sensitized to PBS and challenged with PBS aerosols.

To further define the role of mTOR activation in the effect of EX-527 on allergic airway inflammation, asthmatic mice were intraperitoneally treated with the mTOR inhibitor rapamycin $(4 \mathrm{mg} / \mathrm{kg}) 30 \mathrm{~min}$ prior to $\mathrm{EX}-527$ administration. A total of $24 \mathrm{~h}$ after the last OVA challenge, the mice were anesthetized with an intraperitoneal injection of $1 \%$ pentobarbital sodium (50 mg/kg; Sigma-Aldrich; Merck KGaA) followed by cervical dislocation. Mice were considered dead when respiratory arrest and cardiac arrest were observed, their nerve reflexes disappeared, and muscles relaxed. Mice health and behavior were monitored every day for 28 days, and none of the rats became severely ill or moribund. Fig. 1 shows the treatment regimen through the course of the experiment. BALF was collected for determining cytokine levels and cell counts. The left lungs were preserved and fixed with $10 \%$ formalin for $24 \mathrm{~h}$ at room temperature and then stained with hematoxylin for $5 \mathrm{~min}$ and eosin for $3 \mathrm{~min}$ at room temperature for histopathological analysis under a light microscope (Olympus Corporation). The right lungs were collected and frozen at $-80^{\circ} \mathrm{C}$ for further experiments.

Reagents. OVA and rapamycin were purchased from Sigma-Aldrich (Merck KGaA). ELISA kits for detecting IL-4 [cat. no. KGEHC006(H)-1], IL-13 (cat. no. KGEMC124-1) and interferon (IFN)- $\gamma$ (cat. no. KGEBC101g-1) were purchased from Yufeng Technology Co., Ltd. Rabbit anti-mouse SIRT1 (1:1,000; cat. no. ab110304), microtubule-associated protein 1 light chain $3 \beta$ (LC3B; 1:1,000; cat. no. ab243506) and Beclin-1(1:1,000; cat. no. ab114071) antibodies, and EX-527 (cat. no. ab141506) were purchased from Abcam. Antibodies against total (t)-mTOR (1:1,000; cat. no. 2972) and p-mTOR (1:1,000; cat. no. 5536) were purchased from Cell Signaling Technology, Inc. Anti- $\beta$-actin antibodies (1:5,000; cat. no. AM33096PU-S) were from OriGene Technologies, Inc.

Histological analysis. The left lobe of the lung was fixed in formalin and paraffin embedded. The sections were stained with hematoxylin and eosin for histopathological evaluation under a light microscope (Olympus Corporation).

Collection of BALF. A tracheal tube was inserted for a 1-ml ice-cold PBS lavage, and the obtained BALF was centrifuged at $500 \mathrm{x} \mathrm{g}$ at $4^{\circ} \mathrm{C}$ for $5 \mathrm{~min}$. Next, the supernatant was collected and stored at $-80^{\circ} \mathrm{C}$ for subsequent cytokine measurements. The levels of IL-4, IL-13 and IFN- $\gamma$ were determined by ELISA kits, according to the manufacturer's instructions. The cell pellets were obtained and resuspended in PBS for total cell and eosinophil counts. The slides were fixed and stained with Diff-Quik Stain (cat. no. G1541; Beijing Solarbio Science \& Technology Co., Ltd.) according to the manufacturer's protocol, and differential cell counts were obtained using light microscopic evaluation of 300 cells/slide. Total BALF cells were counted on a haemocytometer.

Western blot analysis. Lung tissue samples were lysed in RIPA Lysis Buffer (Guangzhou Fansi Biotechnology Co., Ltd.). Lysates were centrifuged at $6500 \mathrm{xg}$ at $4^{\circ} \mathrm{C}$ for $15 \mathrm{~min}$, and supernatant was collected and quantified with a BCA protein assay kit (Pierce; Thermo Fisher Scientific, Inc.). $40 \mu \mathrm{g}$ of proteins were separated by $10 \%$ SDS-PAGE and transferred onto a PVDF membrane (cat. no. IPVH00005; EMD Millipore) and blocked with 5\% BSA (Beijing Solarbio Science $\&$ Technology Co., Ltd.) for $1 \mathrm{~h}$ at room temperature. The membranes were probed with polyclonal antibodies against SIRT1, LC3B, Beclin-1, t-mTOR, p-mTOR, and a monoclonal antibody against $\beta$-actin in Tris-buffered saline containing Tween-20 (TBST; Beijing Solarbio Science \& Technology Co., Ltd.) for $2 \mathrm{~h}$ at room temperature. then incubated with goat anti-rabbit secondary antibody in TBST for $1 \mathrm{~h}$ at room temperature. Reactions were developed with SuperSignal West Pico Chemiluminescent Substrate (Pierce; Thermo Fisher Scientific, Inc.), followed by exposure to autoradiographic films. Signals were semi-quantified from scanned films using Quantity One software (v4.6.6, Bio-Rad Laboratories, Inc.).

Statistical analysis. All data were analyzed with SPSS 19.0 software (IBM Corp.). Values are presented as the mean \pm SD 


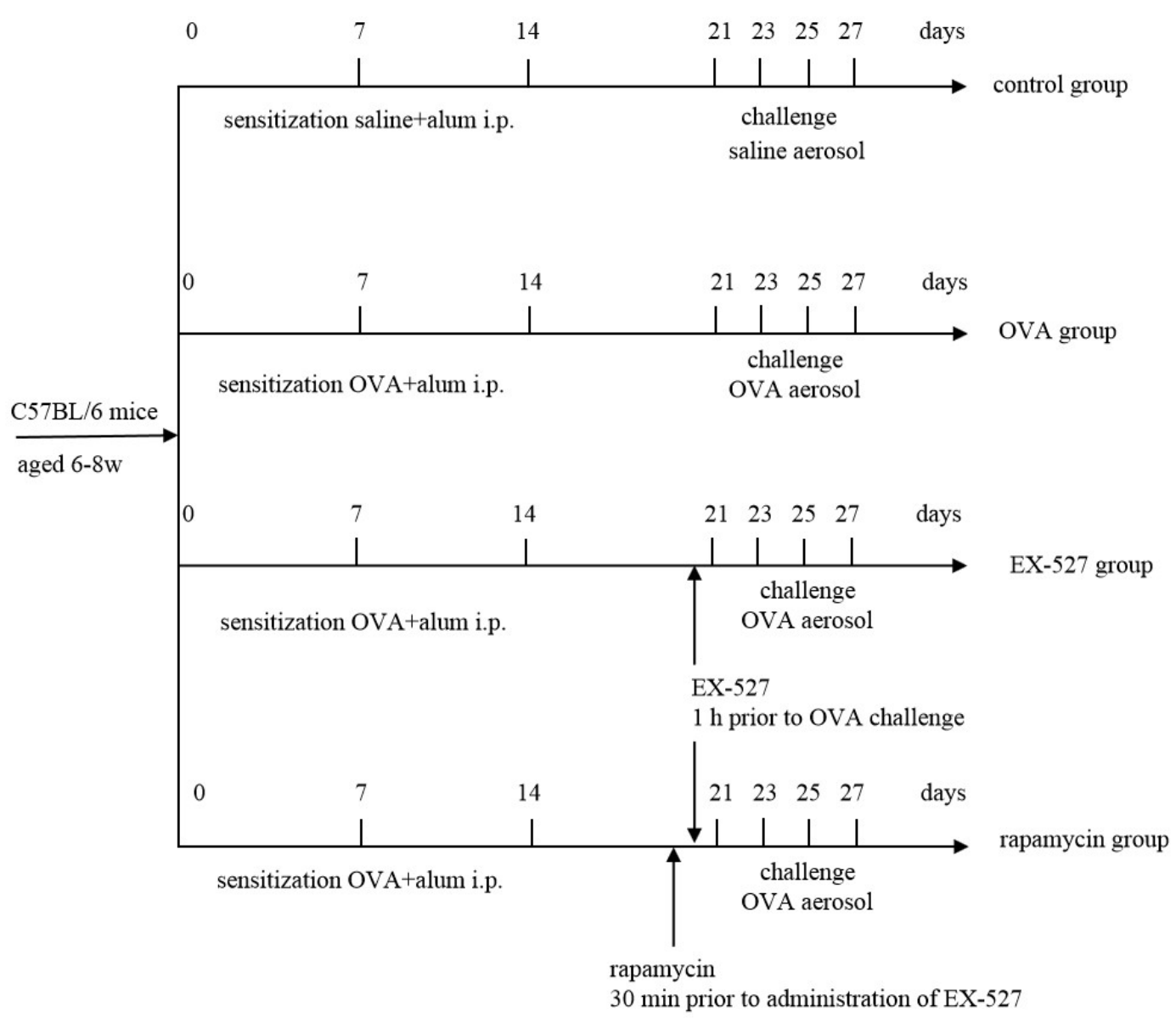

Figure 1. Experimental procedure. C57BL/6 mice were sensitized by intraperitoneal injection of OVA in alum and challenged with $1 \%$ OVA solution (or aerosolized saline) by inhalation. For treatment, EX-527 was administered intraperitoneally $1 \mathrm{~h}$ before the initial OVA challenge. To determine the effects of rapamycin, the latter compound was administered $30 \mathrm{~min}$ before treatment with EX-527. All treatment days are shown. alum, aluminum hydroxide; OVA, ovalbumin; i.p., intraperitoneal injection.

( $\mathrm{n}=8$ mice/group). Data were assessed by one-way ANOVA followed by Tukey's post hoc test. $\mathrm{P}<0.05$ was considered to indicate a statistically significant difference.

\section{Results}

EX-527 alleviates OVA-induced mice airway inflammation, and rapamycin reverses this effect. BALF samples were collected $24 \mathrm{~h}$ after the final OVA challenge. In order to determine the effects of EX-527 on airway inflammation, total cell and eosinophil counts in BALF samples from OVA-induced asthmatic mice were examined. As shown in Fig. 2A, OVA administration significantly increased total cell counts in the BALF compared with the control group $(\mathrm{P}<0.05)$. After EX-527 administration, total cell counts were significantly reduced compared with the OVA group $(\mathrm{P}<0.05$; Fig. 2A). BALF eosinophil levels were consistent with total cell counts. Specifically, OVA treatment resulted in markedly increased eosinophil levels in BALF samples in comparison with the control group $(\mathrm{P}<0.05$; Fig. $2 \mathrm{~A})$. Meanwhile, the EX-527 group exhibited decreased eosinophil levels compared with the OVA treatment group $(\mathrm{P}<0.05$; Fig. $2 \mathrm{~A})$. In order to evaluate the role of mTOR in the EX-527-mediated protective effect on asthma, OVA-induced allergic mice were treated with EX-527 in combination with rapamycin. As shown in
Fig. 2A, rapamycin restored the decreased amounts of total inflammatory cells and eosinophils induced by EX-527 $(\mathrm{P}<0.05)$.

A Th1/Th2 imbalance is believed to play a vital role in asthma pathogenesis, including changes in the levels of Th1 cytokines (IFN- $\gamma$ ) and Th2 cytokines (IL-4 and IL-13) (2). To determine the effects of EX-527 on cytokine release in OVA-induced asthmatic mice, BALF IL-4, IL-13 and IFN- $\gamma$ levels normalized to total protein amounts in BALF were evaluated by ELISA $24 \mathrm{~h}$ after the final OVA challenge. Asthmatic mice exhibited marked increases in IL-4 $(0.77 \pm 0.03$ vs. $0.12 \pm 0.03)$ and IL-13 (0.86 \pm 0.06 vs. $0.23 \pm 0.02)$ levels, and a significant decrease in IFN- $\gamma(0.11 \pm 0.01$ vs. $0.27 \pm 0.03)$ levels $(\mathrm{P}<0.01$; Fig. 2B-D). Treatment with EX-527 resulted in markedly reduced IL-4 $(0.28 \pm 0.03)$ and IL-13 $(0.44 \pm 0.01)$ levels, and significantly increased IFN- $\gamma$ levels $(0.20 \pm 0.02)$ in BALF samples in comparison with the OVA group $(\mathrm{P}<0.05$; Fig. 2B-D). Consistent with the aforementioned results, rapamycin treatment partially reversed the reductions in IL-4 $(\mathrm{P}<0.05$; Fig. 2B) and IL-13 ( $\mathrm{P}<0.05$; Fig. 2C) levels, and the increase in IFN- $\gamma$ levels $(\mathrm{P}<0.05$; Fig. 2D).

Histopathological examination of mouse lungs was performed in order to confirm the inhibitory effect of EX-527 on airway inflammation. The extracted lung tissues exhibited marked infiltration of inflammatory cells in perivascular and 

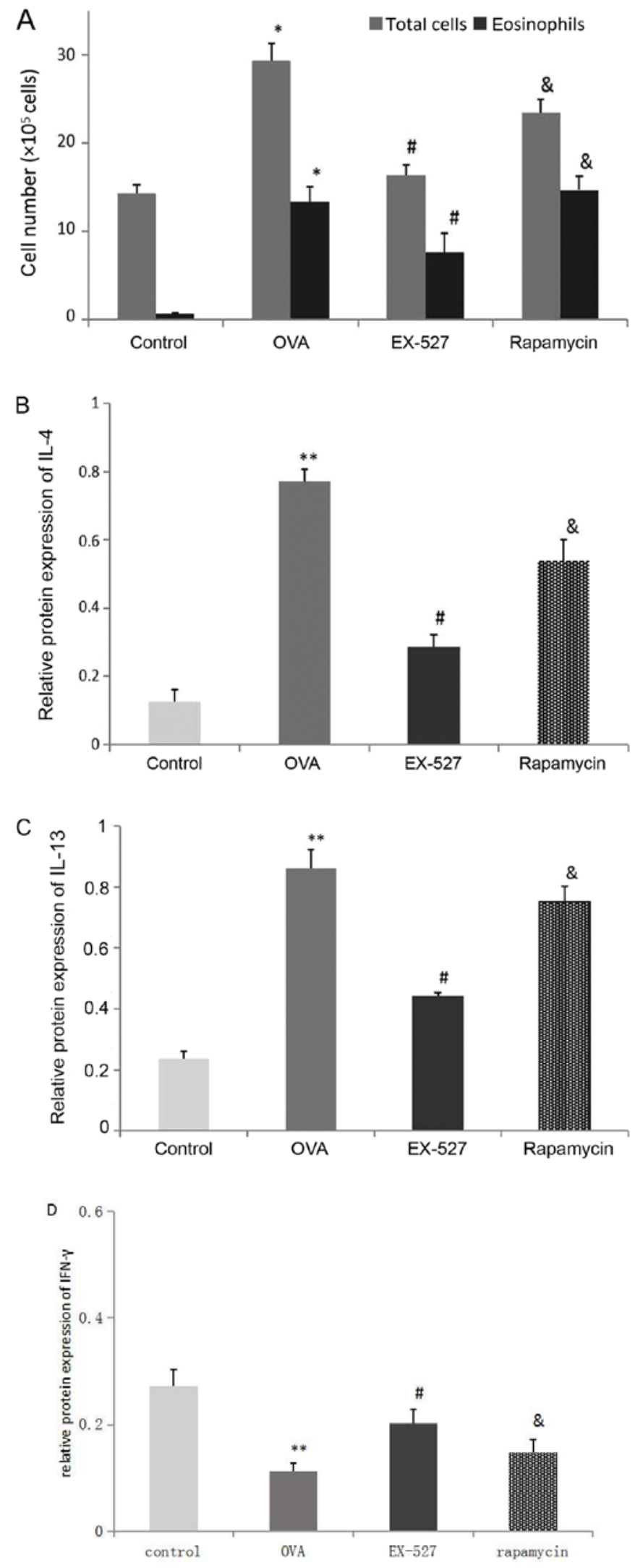

Figure 2. Effects of EX-527 alone or in combination with rapamycin on inflammatory cell counts and cytokine levels in BALF. (A) Cell counts in BALF. (B) IL-4, (C) IL-13 and (D) IFN- $\gamma$ levels in BALF were detected by ELISA $(n=6)$. Control, saline treatment; OVA, OVA sensitization/challenge; EX-527, EX-527 (10 mg/kg) + OVA sensitization/challenge; rapamycin, rapamycin $(4 \mathrm{mg} / \mathrm{kg})+\mathrm{EX}-527(10 \mathrm{mg} / \mathrm{kg})+$ OVA sensitization/challenge. ${ }^{*} \mathrm{P}<0.05$ and ${ }^{* *} \mathrm{P}<0.01$ vs. control group; ${ }^{\#} \mathrm{P}<0.05$ vs. OVA group; ${ }^{\&} \mathrm{P}<0.05$ vs. EX-527 group. BALF, bronchoalveolar lavage fluid; OVA, ovalbumin; IL-, interleukin; IFN-, interferon.
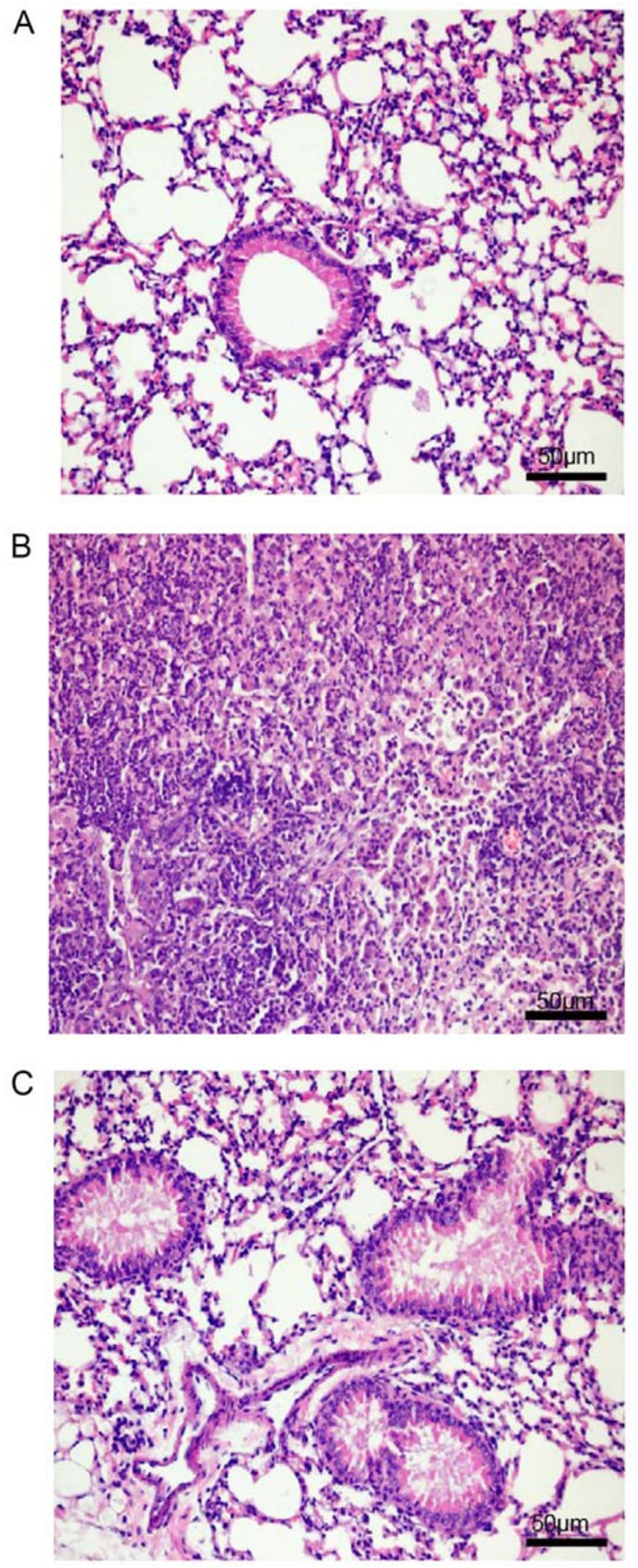

Figure 3. Effects of EX-527 alone or in combination with rapamycin on OVA-induced lung histopathological changes. Lung tissues from each group were stained with hematoxylin and eosin for histological evaluation (magnification, x200). (A) Control; (B) OVA and (C) OVA + EX-527.

peribronchiolar connective tissue samples from the OVA group compared with control mice, and most leukocytes were eosinophils (Fig. 3A and B). Meanwhile, mice treated with EX-527 prior to OVA challenge exhibited markedly decreased inflammatory cell infiltration around the airways and blood vessels (Fig. 3C). As expected, co-administration 


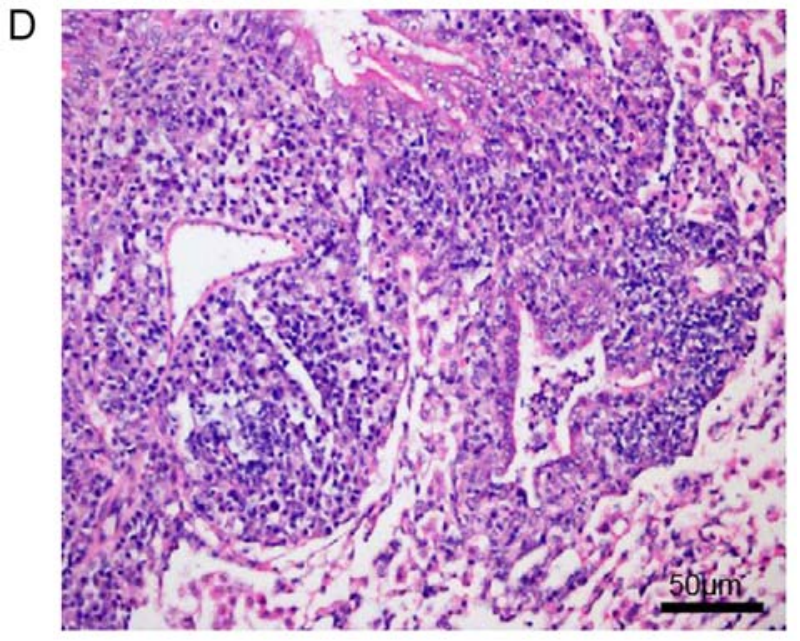

Figure 3. Continued. Effects of EX-527 alone or in combination with rapamycin on OVA-induced lung histopathological changes. Lung tissues from each group were stained with hematoxylin and eosin for histological evaluation (magnification, x200). (D) rapamycin. Control, saline treatment; OVA, OVA sensitization/challenge; EX-527, EX-527 (10 mg/kg) + OVA sensitization/challenge; rapamycin, rapamycin $(4 \mathrm{mg} / \mathrm{kg})+\mathrm{EX}-527(10 \mathrm{mg} / \mathrm{kg})+$ OVA sensitization/challenge. OVA, ovalbumin.

of rapamycin partly abolished the anti-inflammatory effects of EX-527 (Fig. 3D). These findings indicated that the beneficial effects of EX-527 on OVA-induced allergic mice may be inhibited by rapamycin.

Anti-inflammatory effects of EX-527 are associated with suppressed autophagy. Because EX-527 is considered a selective SIRT1 inhibitor, the study next assessed whether its beneficial effects are associated with SIRT1 inhibition. Western blot analysis indicated that OVA exposure resulted in increased SIRT1 expression, which was significantly reversed by treatment with EX-527 (Fig. 4A). These data suggested that EX-527 suppressed airway inflammation in asthmatic mice by inhibiting SIRT1, since EX-527 is a well-known specific inhibitor of SIRT1-1 (22). LC3B, a mammalian homolog of yeast Atg8, is comprised of two forms: LC3-I (18 kDa) and LC3-II (16 kDa). During autophagy the cytoplasmic form LC3-I is processed and recruited to autophagosomes, where LC3-II is generated by site-specific proteolysis near the C-terminus (24). The ratio of LC3-II to LC3-I is commonly used to analyze autophagic activity, of which Beclin-1 is also considered a hallmark (17). Furthermore, it has been reported that autophagy suppression may inhibit airway inflammation in allergic mice (16). Therefore, the present study assessed whether EX-527 exerted anti-inflammatory effects by suppressing autophagy. In this study, the LC3-II/LC3-I ratio was significantly increased in mice in the OVA group compared with the control group $(\mathrm{P}<0.01)$, and this upregulation was alleviated by EX-527 administration ( $\mathrm{P}<0.05$; Fig. 4B). Similarly, Beclin-1 expression was increased by OVA and decreased by EX-527 (Fig. 4C). Thus, the beneficial effects of EX-527 might be attributed to LC3-II and Beclin-1 suppression. These findings indicated that autophagy was upregulated in OVA-induced allergic asthmatic mice but alleviated by SIRT1 inhibition.
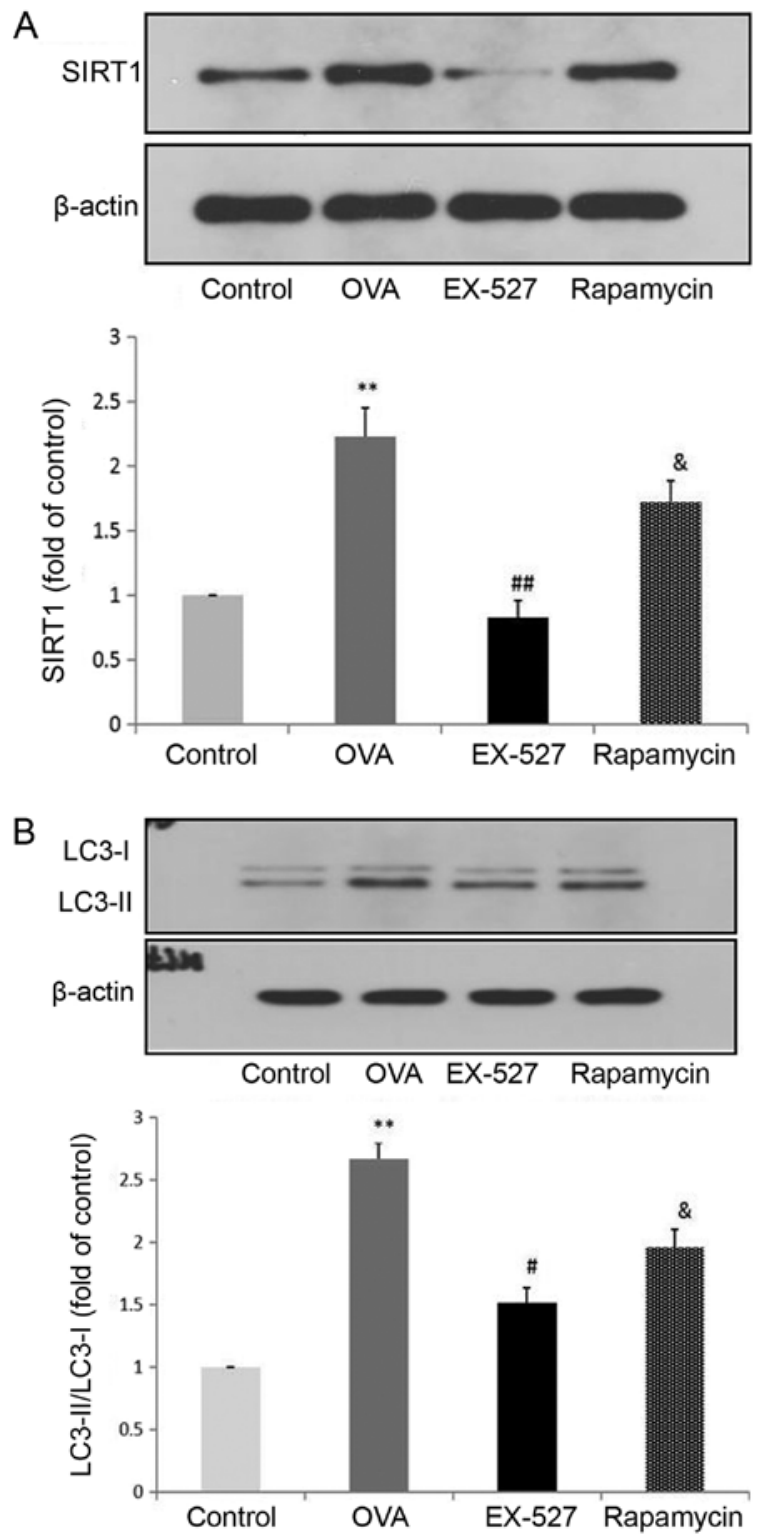

Figure 4. EX-527 suppresses OVA-induced autophagy, and this effect is abolished by rapamycin. Western blot analysis of (A) SIRT1 and (B) LC3-II/LC3-I.

SIRT1 regulates autophagy through the mTOR signaling pathway. mTOR activation was induced after treatment with EX-527 in OVA-stimulated mice. To investigate whether mTOR-mediated autophagy was involved in the protective effect of EX-527 on OVA-induced allergic airway inflammation, the OVA group was administered rapamycin $30 \mathrm{~min}$ prior to treatment with EX-527. Subsequently, SIRT1, LC3-II/LC3-I, Beclin-1 and p-mTOR expression levels were determined by western blotting. Notably, rapamycin significantly reversed the inhibited autophagic flux induced by EX-527, as reflected by increased SIRT1 (Fig. 4A; P<0.05), LC3-II/LC3-I (Fig. 4B; $\mathrm{P}<0.05$ ) and Beclin-1 expression (Fig. 4C; P<0.05), and decreased levels of p-mTOR (Fig. 4D; $\mathrm{P}<0.05)$. These results suggested that the inhibitory effect EX-527 on autophagy was significantly suppressed by rapamycin, which indicated that EX-527 inhibited autophagy through mTOR signaling suppression. 

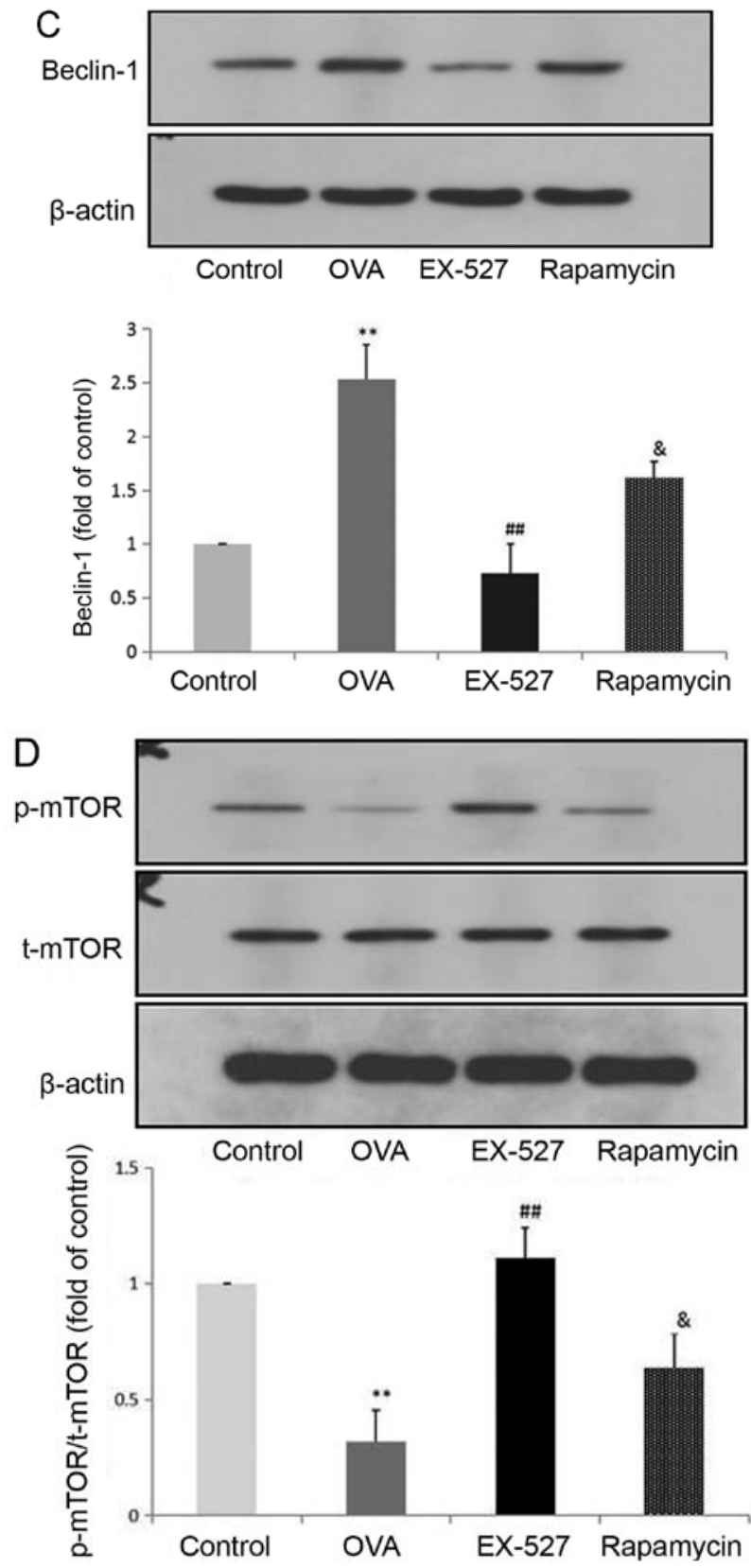

Figure 4. Continued. EX-527 suppresses OVA-induced autophagy, and this effect is abolished by rapamycin. Western blot analysis of (C) Beclin-1 and (D) $\mathrm{p}$-mTOR/t-mTOR. $\beta$-actin was used as a loading control. Control, saline treatment; OVA, OVA sensitization/challenge; EX-527, EX-527 $(10 \mathrm{mg} / \mathrm{kg})+$ OVA sensitization/challenge; rapamycin, rapamycin $(4 \mathrm{mg} / \mathrm{kg})$ + EX-527 $(10 \mathrm{mg} / \mathrm{kg})+$ OVA sensitization/challenge. ${ }^{* *} \mathrm{P}<0.01$ vs. control group; ${ }^{\#} \mathrm{P}<0.05$ and ${ }^{\# \#} \mathrm{P}<0.01$ vs. OVA group; ${ }^{\circledR} \mathrm{P}<0.05$ vs. EX-527 group. OVA, ovalbumin; SIRT1, sirtuin 1; LC3, microtubule-associated protein 1 light chain $3 \beta$; mTOR, mammalian target of rapamycin; p, phosphorylated; $\mathrm{t}$, total.

\section{Discussion}

In the present study, SIRT1 inhibition by EX-527 effectively alleviated airway inflammation in OVA-induced asthmatic mice through suppression of autophagic flux; this effect was mediated by mTOR pathway activation. It is known that loss of balance between Th1 and Th2 plays a critical role in asthma pathophysiology (25). A Th1/Th2 imbalance can be triggered by changes in the levels of IFN- $\gamma$ and IL-4 secreted by Th1 and Th2 cells, respectively (26). In the OVA-induced mouse model of asthma, allergen exposure has been reported to produce eosinophilia, airway hyperresponsiveness, mucus hypersecretion and a Th2-dominated response, such as increased IL-4 and IL-13 in plasma and BALF (27). Previous findings indicated that suppression of SIRT1 prevented asthma progression, alleviated airway hyperresponsiveness, decreased inflammatory cell numbers around the airways, and decreased IL-4, IL-5 and IL-13 levels in BALF in OVA-inhaled mice (8). The present results indicated that compared with OVA-induced allergic mice, EX-527-treated mice had significantly fewer total inflammatory cells and eosinophils, with decreased levels of the Th2 cytokines IL-4 and IL-13, but increased IFN- $\gamma$ in BALF. Administration of rapamycin to OVA-sensitized/challenged mice significantly reversed the anti-inflammatory effects of EX-527.

It was previously reported that increased expression of SIRT1 induced autophagy, and SIRT1 activation could enhance autophagy, while its inhibition suppressed autophagy in vascular adventitial fibroblasts (28). Another study revealed that SIRT1 protected cardiomyocytes from hypoxic stress by promoting autophagic flux induced by AMPK activation (29). SIRT1 has also been reported to be involved in the pathogenesis of OVA-induced asthma in mice, and airway inflammation and hyperresponsiveness were attenuated after its inhibition (8). In addition, autophagy in smooth muscle cells was enhanced in OVA-induced allergic mice and reducing cell autophagy significantly attenuated OVA-induced airway inflammation (17). To further determine whether SIRT1 inhibition suppressed inflammation in allergic asthma by autophagy suppression, mice were intraperitoneally injected with EX-527 before challenge with OVA. It was revealed that LC3-II/LC3-I and Beclin-1 expression levels were increased in the OVA group but were significantly reduced after EX-527 administration.

mTOR is an evolutionarily conserved serine/threonine protein kinase, which acts as a central regulator of cell proliferation, cell growth, survival, autophagy and transcription $(30,31)$. mTOR activation has been shown to reduce inflammation in an OVA-induced allergic mouse model (16) and autophagy is known to be controlled by the mTOR signaling pathway (19). Furthermore, mTOR levels were decreased in the lungs of allergic mice, whereas mTOR activation inhibited allergic airway inflammation via suppressed autophagy (16). Therefore, mTOR could represent a pivotal modulator of asthma development. Meanwhile, the mTOR and SIRT1 signaling pathways have been shown to be closely integrated (20). SIRT1 has been reported to ameliorate systemic sclerosis via inhibition of mTOR phosphorylation (32). It was subsequently assessed whether mTOR may be involved in the anti-inflammatory effects of EX-527 observed in OVA-induced asthmatic mice. The present results indicated that asthmatic mice treated with EX-527 exhibited relieved OVA-induced mTOR inhibition and autophagy enhancement. Co-administration of EX-527 with rapamycin reversed the suppression of autophagy, and partly abolished the anti-inflammatory effects of EX-527. In addition, significantly increased p-mTOR expression, and decreased LC3-II/LC3-I and Beclin-1 levels after treatment with EX-527 were reversed by treatment with rapamycin. These findings suggested that the inhibitory effects of EX-527 on autophagy may be significantly suppressed by rapamycin and might be achieved by inhibiting mTOR activation. 
The present study revealed that EX-527 administration in asthmatic mice downregulated the expression of Th2-related cytokines IL-4 and IL-13 and upregulated the Th1-type cytokine IFN- $\gamma$. EX-527 significantly reduced leukocyte and eosinophil infiltration into the airways and lung tissue of OVA-induced asthmatic mice. Similarly, LC3-II/LC3-I and Beclin-1 expression were decreased after EX-527 administration. Further evidence indicated that rapamycin reversed the inhibitory effects of EX-527 on airway inflammation and autophagy influx in OVA-induced asthmatic mice.

Taken together, these findings indicated that EX-527 alleviated airway inflammation in asthmatic mice, likely through modulation of the mTOR pathway. Therefore, mTOR signaling could be targeted for asthma control. Further related studies are warranted.

As a notable limitation of the present study, there wasn't an EX-527-only group. Furthermore, transmission electron microscopy could be applied to assess autophagy in future studies. Finally, the present study was performed in a mouse model, which may not completely recapitulate the clinical situation. Therefore, clinical trials are also warranted to determine the translational potential of the present findings.

In conclusion, the present study demonstrated that EX-527 effectively inhibited allergen-induced airway inflammation in an asthma model via mTOR-mediated autophagy.

\section{Acknowledgements}

Not applicable.

\section{Funding}

The present study was supported by from the National Natural Science Foundation of China (grant no. 81800030), the Natural Science Foundation of Shanxi Province (grant no. 2018JQ8034) and the Xi'an science and technology project (grant no. 2017113SF/ YX007).

\section{Availability of data and materials}

The datasets used and/or analyzed during the current study are available from the corresponding author on reasonable request.

\section{Authors' contributions}

XS and YL contributed to the conception and design of the study and gave final approval of the final version to be published. YW performed the majority of the study, data analysis and drafted the manuscript. YH provided pathological assistance and was involved in the data analysis. WL contributed to interpretation of the data and analyses. All authors read and approved the final manuscript.

\section{Ethics approval and consent to participate}

All experimental protocols were approved by the Medical Ethics Committee of The Second Affiliated Hospital of Xi'an Jiaotong University (approval no. 2019043).

\section{Patient consent for publication}

Not applicable.

\section{Competing interests}

The authors declare that they have no competing interests.

\section{References}

1. Tang L, Chen Q, Meng Z, Sun L, Zhu L, Liu J, Hu J, Ni Z and Wang X: suppression of sirtuin-1 increases IL- 6 expression by activation of the Akt pathway during allergic asthma. Cell Physiol Biochem 43: 1950-1960, 2017.

2. Ding F, Fu Z and Liu B: Lipopolysaccharide exposure alleviates asthma in mice by regulating Th1/Th2 and Treg/Th17 balance. Med Sci Monit 24: 3220-3229, 2018.

3. Islam S, Abiko Y, Uehara O and Chiba I: Sirtuin 1 and oral cancer. Oncol Lett 17: 729-738, 2019.

4. Kida Y and Goligorsky MS: Sirtuins, cell senescence, and vascular aging. Can J Cardiol 32: 634-641, 2016.

5. Lin Z and Fang D: The roles of SIRT1 in cancer. Genes Cancer 4: 97-104, 2013.

6. Rahman S and Islam R: Mammalian Sirt1: Insights on its biological functions. Cell Commun Signal 9: 11, 2011.

7. Potteti HR, Rajasekaran S, Rajamohan SB, Tamatam CR, Reddy NM and Reddy SP: Sirtuin 1 promotes hyperoxia-induced lung epithelial cell death independent of NF-E2-related factor 2 activation. Am J Respir Cell Mol Biol 54: 697-706, 2016.

8. Kim SR, Lee KS, Park SJ, Min KH, Choe YH, Moon H, Yoo WH, Chae HJ, Han MK and Lee YC: Involvement of sirtuin 1 in airway inflammation and hyperresponsiveness of allergic airway disease. J Allergy Clin Immunol 125: 449-460.e4, 2010.

9. Wang Y, Li D, Ma G, Li W, Wu J, Lai T, Huang D, Zhao X, Lv Q, Chen M and Wu B: Increases in peripheral SIRT1: A new biological characteristic of asthma. Respirology 20: 1066-1072, 2015.

10. Liu Y, Yang F, Zou S and Qu L: Rapamycin: A bacteria-derived immunosuppressant that has anti-atherosclerotic effects and its clinical application. Front Pharmacol 9: 1520, 2019.

11. Saxton RA and Sabatini DM: mTOR signaling in growth, metabolism, and disease. Cell 168: 960-976, 2017.

12. Lin J, Huo X and Liu X: 'mTOR signaling pathway': A potential target of curcumin in the treatment of spinal cord injury. Biomed Res Int 2017: 1634801, 2017.

13. Salido-Vallejo R, Garnacho-Saucedo G and Vélez A: Elucidation of the mTOR pathway and therapeutic applications in dermatology. Actas Dermosifiliogr 107: 379-390, 2016 (In English, Spanish).

14. Perl A: Activation of mTOR (mechanistic target of rapamycin) in rheumatic diseases. Nat Rev Rheumatol 12: 169-182, 2016.

15. Wang Y, Liu J, Zhou JS, Huang HQ, Li ZY, Xu XC, Lai TW, $\mathrm{Hu}$ Y, Zhou HB, Chen HP, et al: MTOR suppresses cigarette smoke-induced epithelial cell death and airway inflammation in chronic obstructive pulmonary disease. J Immunol 200: 2571-2580, 2018.

16. Zou H, Wang LX, Wang M, Cheng C, Li S, Shen Q, Fang L and Liu R: MTOR-mediated autophagy is involved in the protective effect of ketamine on allergic airway inflammation. J Immunol Res 2019: 5879714, 2019.

17. Cheng Z, Wang X, Dai L, Jia L, Jing X, Liu Y, Wang H, Li P, An L and Liu M: Suppression of microRNA-384 enhances autophagy of airway smooth muscle cells in asthmatic mouse. Oncotarget 8: 67933-67941, 2017.

18. Farooq MB and Walsh GM: Autophagy and asthma. Clin Exp Allergy 46: 7-9, 2016.

19. Urbanska M, Gozdz A, Swiech LJ and Jaworski J: Mammalian target of rapamycin complex 1 (mTORC1) and 2 (mTORC2) control the dendritic arbor morphology of hippocampal neurons. J Biol Chem 287: 30240-30256, 2012.

20. Ma L, Dong W, Wang R, Li Y, Xu B, Zhang J, Zhao Z and Wang Y: Effect of caloric restriction on the SIRT1/mTOR signaling pathways in senile mice. Brain Res Bull 116: 67-72, 2015.

21. Guo W, Qian L, Zhang J, Zhang W, Morrison A, Hayes P, Wilson S, Chen T and Zhao J: Sirtl overexpression in neurons promotes neurite outgrowth and cell survival through inhibition of the mTOR signaling. J Neurosci Res 89: 1723-1736, 2011. 
22. Huang J, Tian R, Yang Y, Jiang R, Dai J, Tang L and Zhang L: The SIRT1 inhibitor EX-527 suppresses mTOR activation and alleviates acute lung injury in mice with endotoxiemia. Innate Immun 23: 678-686, 2017.

23. Zhou X, Fan LX, Sweeney WE Jr, Denu JM, Avner ED and Li X: Sirtuin 1 inhibition delays cyst formation in autosomal-dominan polycystic kidney disease. J Clin Invest 123: 3084-3098, 2013.

24. Kang R, Tang D, Lotze MT and Zeh Iii HJ: Autophagy is required for IL-2-mediated fibroblast growth. Exp Cell Res 319: 556-565, 2013.

25. Mushaben EM, Kramer EL, Brandt EB, Khurana Hershey GK and Le Cras TD: Rapamycin attenuates airway hyperreactivity, goblet cells, and IgE in experimental allergic asthma. J Immunol 187: 5756-5763, 2011

26. Shao YY, Zhou YM, Hu M, Li JZ, Chen CJ, Wang YJ, Shi XY, Wang WJ and Zhang TT: The anti-allergic rhinitis effect of traditional Chinese medicine of shenqi by regulating mast cell degranulation and Th1/Th2 cytokine balance. Molecules 22: E504, 2017.

27. Lee M, Kim S, Kwon OK, Oh SR, Lee HK and Ahn K: Anti-inflammatory and anti-asthmatic effects of resveratrol, a polyphenolic stilbene, in a mouse model of allergic asthma. Int Immunopharmacol 9: 418-424, 2009.
28. Wang WR, Li TT, Jing T, Li YX, Yang XF, He YH, Zhang W, Lin R and Zhang JY: SIRT1 regulates the inflammatory response of vascular adventitial fibroblasts through autophagy and related signaling pathway. Cell Physiol Biochem 41: 569-582, 2017.

29. Luo G, Jian Z, Zhu Y, Zhu Y, Chen B, Ma R, Tang F and Xiao Y: Sirt1 promotes autophagy and inhibits apoptosis to protect cardiomyocytes from hypoxic stress. Int J Mol Med 43: 2033-2043, 2019.

30. Tan FH, Bai Y, Saintigny P and Darido C: mTOR signalling in head and neck cancer: Heads up. Cells 8: E333, 2019.

31. Rabanal-Ruiz Y, Otten EG and Korolchuk VI: mTORC1 as the main gateway to autophagy. Essays Biochem 61: 565-584, 2017.

32. Zhu X, Chu H, Jiang S, Liu Q, Liu L, Xue Y, Zheng S, Wan W, Qiu J, Wang J and Zou H: Sirt1 ameliorates systemic sclerosis by targeting the mTOR pathway. J Dermatol Sci 87: 149-158, 2017.

(i) (9) This work is licensed under a Creative Commons Attribution-NonCommercial-NoDerivatives 4.0 International (CC BY-NC-ND 4.0) License. 